\title{
Wikipedia Entities as Rendezvous across Languages: Grounding Multilingual Language Models by Predicting Wikipedia Hyperlinks
}

\author{
Iacer Calixto $^{1,2} \quad$ Alessandro Raganato $^{3} \quad$ Tommaso Pasini $^{4, *}$ \\ ${ }^{1}$ Center for Data Science, New York University ${ }^{2}$ ILLC, University of Amsterdam \\ ${ }^{3}$ Department of Digital Humanities, University of Helsinki, Finland \\ ${ }^{4}$ Department of Computer Science, University of Copenhagen \\ iacer.calixto@nyu.edu, alessandro.raganato@helsinki.fi, \\ tommaso.pasini@di.ku.dk
}

\begin{abstract}
Masked language models have quickly become the de facto standard when processing text. Recently, several approaches have been proposed to further enrich word representations with external knowledge sources such as knowledge graphs. However, these models are devised and evaluated in a monolingual setting only. In this work, we propose a languageindependent entity prediction task as an intermediate training procedure to ground word representations on entity semantics and bridge the gap across different languages by means of a shared vocabulary of entities. We show that our approach effectively injects new lexicalsemantic knowledge into neural models, improving their performance on different semantic tasks in the zero-shot crosslingual setting. As an additional advantage, our intermediate training does not require any supplementary input, allowing our models to be applied to new datasets right away. In our experiments, we use Wikipedia articles in up to 100 languages and already observe consistent gains compared to strong baselines when predicting entities using only the English Wikipedia. Further adding extra languages lead to improvements in most tasks up to a certain point, but overall we found it non-trivial to scale improvements in model transferability by training on ever increasing amounts of Wikipedia languages.
\end{abstract}

\section{Introduction}

Pretrained Multilingual Masked Language Models (MMLMs) such as mBERT (Devlin et al., 2019), XLM-R (Conneau et al., 2020) and their variants have achieved state-of-the-art results across diverse natural language understanding tasks. Typically, a MMLM model is pretrained on very large amounts of raw text in different languages using the masked language modelling (MLM) objective and is further finetuned on (usually limited amounts of) task data.

\footnotetext{
*Work carried out while at the University of Rome "La Sapienza".
}

In the zero-shot crosslingual setting, which is our focus in this paper, a MMLM is finetuned on the target task using data in a single language (e.g., English) and is evaluated on the same task but in different languages (e.g., non-English languages).

We introduce the multilingual Wikipedia hyperlink prediction objective to contextualise words in a text with entities and concepts from an external knowledge source by using Wikipedia articles in up to 100 languages. Hyperlink prediction is a knowledge-rich task designed to (1) inject semantic knowledge from Wikipedia entities and concepts into the MMLM token representations, and (2) with a similar motivation as the translated language modelling loss of Conneau and Lample (2019), i.e., to inject explicit language-independent knowledge into a model trained via self-supervised learning, but in our case without parallel data. We devise a training procedure where we mask out hyperlinks in Wikipedia articles and train the MMLM to predict the hyperlink identifier similarly to standard MLM but using a "hyperlink vocabulary" of 250k concepts shared across languages.

We use the state-of-the-art MMLM XLM-Rlarge (Conneau et al., 2020) and show that by adding an add-on training step using Wikipedia hyperlink prediction we consistently improve several zero-shot crosslingual natural language understanding tasks across a diverse array of languages: crosslingual Word Sense Disambiguation in 18 languages including English (XL-WSD; Pasini et al., 2021); the crosslingual Word-in-Context task (XLWiC; Raganato et al., 2020) in 12 non-English languages; and in 7 tasks from the XTREME benchmark (Hu et al., 2020) in up to 40 languages.

Recently, Zhang et al. (2019, ERNIE) and Peters et al. (2019, KnowBERT) devised different methods to incorporate entities from external knowledge graphs into masked language model (LM) training. Since then, several works followed (Wang et al., 2021; Sun et al., 2020; Xiong et al., 2020; Yamada 


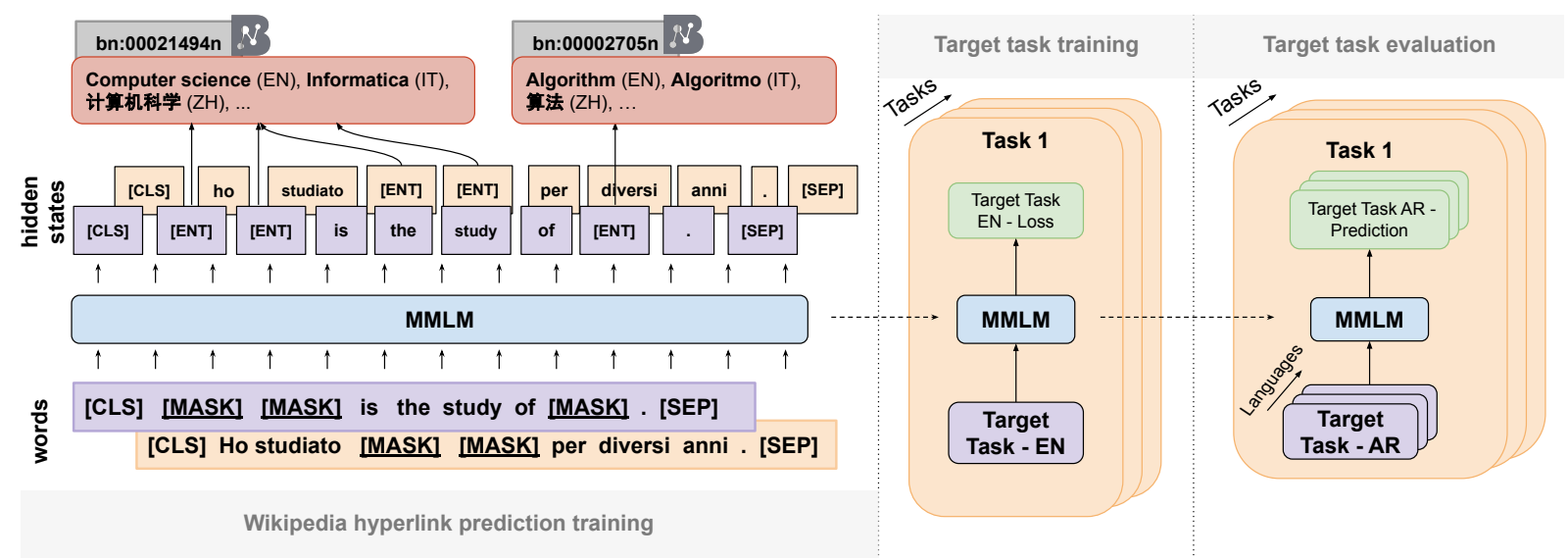

Figure 1: We finetune a pretrained MMLM using multilingual Wikipedia hyperlink prediction, then further train a model on a set of target tasks in English and evaluate on non-English data (i.e., zero-shot crosslingual setting).

et al., 2020) showing increasingly better performance than masked LMs that rely on information from raw text only. Nevertheless, all these methods were proposed for a single language ${ }^{1}$ and cannot be easily applied to transfer learning in a zero-shot crosslingual setting.

\section{Approach}

Notation Let $\boldsymbol{x}_{1: m}=\operatorname{MMLM}\left(x_{1: m}\right)$ be contextualised word representations for some input text $x_{1: m}$ with $m$ words, and computed with a pretrained MMLM. Let $\boldsymbol{x}_{n: k}(n \geq 1, k \leq m)$ be a subsequence of contextualised word representations of a single hyperlink $x_{n: k}$ consisting of $k-n$ words. In our working example we use a single hyperlink $x_{n: k}$ for simplicity, but in practice there may be multiple hyperlinks in the input $x_{1: m}$.

Data We download and preprocess Wikipedia articles in 100 languages, and extract all hyperlinks in the text. We use BabelNet (Navigli and Ponzetto, 2010) - a large multilingual knowledge base comprising WordNet, Wikipedia, and many other resources - to map Wikipedia articles in different languages about the same subject onto unique identifiers. For instance, regardless of their language all "computer science" articles are mapped to the same identifier $h_{t}$, in this case bn: 00021494 n. $^{2}$ After each article is mapped to a single identifier, we create prediction targets for every hyperlink by using the identifier of its referenced article. See Appendix A for more details.

\footnotetext{
${ }^{1}$ Mostly English, except for Sun et al. (2020) where Mandarin is also used in a monolingual setting.

${ }^{2}$ https : / / babelnet. org/ synset? word=bn : $00021494 \mathrm{n} \& \mathrm{l}$ ang $=\mathrm{EN}$
}

Wikipedia Hyperlink Prediction Our main goal is to use the rich semantic knowledge contained in the multilingual Wikipedias' structure to improve language model pretraining. Our approach can be seen as intermediate-task training (Phang et al., 2018, 2020) where we use Wikipedias' hyperlinks as labelled data to further finetune a pretrained MMLM model before training it one last time in the actual target task of interest. Motivated by recent studies on pretrained language encoders demonstrating that semantic features are highlighted in higher layers (Raganato and Tiedemann, 2018; Jawahar et al., 2019; Cui et al., 2020; Rogers et al., 2021), we further train only the last two layers of the MMLM. Moreover, similarly to the MLM procedure, we replace the hyperlink tokens $x_{n: k}$ by the [MASK] token or by a random token $80 \%$ and $10 \%$ of the time, respectively (Devlin et al., 2019).

Since the number of Wikipedia articles is very large, we only consider the most frequent $250 \mathrm{k}$ referenced articles $h_{t}$ as possible hyperlinks in our model and we use the adaptive softmax activation function to speed-up training (Grave et al., 2017). Our objective allows us to consider textentity alignments during training only. At prediction time, instead, we simply feed the model with raw text with no need of precomputed alignments. This makes our model easy to use and to adapt to many different scenarios. For more details on the model architectures and objective, see Appendix B.

\section{Experimental Setup}

We use XLM-R-large (Conneau et al., 2020) as our MMLM, which is pretrained on a large volume 
of raw multilingual corpora using MLM training.

\subsection{Models}

We propose three different model architectures which differ in how the input to the hyperlink classification head is computed. In Token we use the vector representation of each token in the hyperlink text $\boldsymbol{x}_{i}, i \in[n, k]$ as input to the prediction head. In Concat CLS we use the concatenation $\left[\boldsymbol{x}_{i} ; \boldsymbol{x}_{\mathrm{CLS}}\right]$ of the representation of each word in the hyperlink $\boldsymbol{x}_{i}, i \in[n, k]$ with the [CLS ] token representation as input to the prediction head. Finally, in Replace CLS the input to the prediction head is the representation of each word in the hyperlink $\boldsymbol{x}_{i}, i \in[n, k]$ with probability $p_{r}$ or the [CLS ] token representation $\boldsymbol{x}_{\mathrm{CLS}}$ with probability $1-p_{r}$. More details on the architectures in Appendix B.1.

\subsection{Methodology}

We follow a sequential, three steps approach to training and evaluating our models. We first finetune the pretrained MMLM on the Wikipedia hyperlink prediction task, then finetune again this time on the target-task training data in English, and finally evaluate the model on non-English target-task evaluation data in a zero-shot crosslingual setting (see Figure 1). We use Wikipedia articles in different sets of languages (Section 3.3) and experiment with many diverse target tasks (Section 3.4).

\subsection{Wikipedia Languages}

We experiment using only English (Wiki EN), 15 different languages (Wiki 15), or 100 Wikipedia languages (Wiki 100). By doing that, i) we include a monolingual albeit resource-rich baseline (Wiki EN), ii) we investigate the impact of including a varied mixture of languages from different families (Wiki 15), and iii) we also experiment if going massively multilingual has a noticeable impact on crosslingual transferability (Wiki 100).

\subsection{Target Tasks}

Word Sense Disambiguation We follow the zero-shot crosslingual setting of Pasini et al. (2021, XL-WSD), which includes 17 languages plus English, i.e., we train on the English SemCor (Miller et al., 1993) dataset merged with the Princeton WordNet Gloss corpus ${ }^{3}$ and test on all available languages (Miller et al., 1993; Raganato et al., 2017; Edmonds and Cotton, 2001; Snyder and Palmer,

\footnotetext{
${ }^{3}$ http://wordnetcode.princeton.edu/ glosstag.shtml
}

2004; Pradhan et al., 2007; Navigli et al., 2007; Agirre et al., 2010; Navigli et al., 2013; Moro and Navigli, 2015; Pociello et al., 2008; Simov and Osenova, 2010; Benítez et al., 1998; Huang et al., 2010; Raffaelli et al., 2008; Pedersen et al., 2009; Postma et al., 2016; Vider and Orav, 2002; Guinovart, 2011; Miháltz et al., 2008; Isahara et al., 2008; Yoon et al., 2009; Fišer et al., 2012).

Word-in-Context We use the crosslingual Wordin-Context dataset (XL-WiC; Raganato et al., 2020) with data in 12 diverse languages. The task is to predict whether an ambiguous word that appears in two different sentences share the same meaning. We finetune the model on the English WiC (Pilehvar and Camacho-Collados, 2019) dataset and evaluate on the $12 \mathrm{XL}-\mathrm{WiC}$ languages.

XTREME The XTREME (Hu et al., 2020) evaluation suite contains diverse tasks in up to 40 different languages. We perform crosslingual evaluation on: question answering (XQuAD; MLQA; TyDiQA; Artetxe et al., 2020; Lewis et al., 2020; Clark et al., 2020), natural language inference (XNLI; Conneau et al., 2018), paraphrase detection (PAWS-X; Yang et al., 2019), part-of-speech tagging (POS; Nivre et al., 2018), and named entity recognition (NER; Pan et al., 2017). As standard in the two unsupervised sentence retrieval tasks, BUCC (Zweigenbaum et al., 2018), and Tatoeba (Artetxe and Schwenk, 2019), XLM-R is tested considering the output of its 14-th layer, which, however, is not tuned during our intermediate task. We therefore do not report results on these tasks. ${ }^{4}$

Task Architectures Across all the tasks, we finetune transformer-based models by adding a classification head for each task. ${ }^{5}$

\section{Results and Discussion}

Results on XL-WSD and XL-WiC tasks (Tables 1 and 2) suggest that our models have a better grasp of word-level semantics than XLM-R, which does not have explicit semantic signals during its pretraining. This is consistent across languages and hyperlink prediction architectures, also when compared to the baseline XLM-R additionally finetuned using MLM training on in-domain Wikipedia data. Our best models outperform the baselines in both tasks by several points. Interestingly, training on

\footnotetext{
${ }^{4}$ More details in Appendix B.2.

${ }^{5}$ Details in Appendix B.1.1 (XL-WSD), B.1.2 (XL-WiC), and B.1.3 (XTREME).
} 


\begin{tabular}{|c|c|c|c|c|c|c|c|c|c|c|c|c|c|}
\hline & \multirow[b]{2}{*}{ XLM-R } & \multicolumn{4}{|c|}{ WIKI EN } & \multicolumn{4}{|c|}{ WIKI 15} & \multicolumn{4}{|c|}{ WIKI 100} \\
\hline & & +MLM & $+T$ & $+\mathrm{C}$ & $+\mathbf{R}$ & +MLM & $+T$ & $+\mathrm{C}$ & $+\mathbf{R}$ & $+\mathrm{MLM}$ & $+\mathrm{T}$ & $+\mathrm{C}$ & $+\mathbf{R}$ \\
\hline $\mathbf{E N}_{\mathrm{ALL}}$ & 77.7 & 77.4 & 76.8 & 78.4 & $\underline{78.5}$ & 77.6 & 78.5 & $\underline{78.7}$ & 78.5 & 77.4 & 78.4 & $\underline{78.6}$ & 78.3 \\
\hline BG & 72.0 & 71.9 & 72.1 & $\underline{72.6}$ & 70.8 & 71.7 & 73.3 & $\underline{73.5}$ & 73.1 & 71.8 & 72.9 & 73.2 & $\underline{73.4}$ \\
\hline CA & 50.0 & 49.5 & 52.7 & $\underline{52.9}$ & 50.8 & 49.9 & 54.0 & 53.7 & $\underline{54.3}$ & 50.1 & 54.6 & 52.7 & 54.7 \\
\hline DA & 80.6 & 80.4 & $\underline{81.7}$ & $\underline{81.7}$ & 79.9 & 80.6 & 82.4 & $\underline{82.5}$ & 82.4 & 80.7 & 82.4 & $\underline{82.8}$ & 82.1 \\
\hline DE & 83.2 & 83.6 & 83.6 & $\underline{84.1}$ & 83.9 & 83.3 & 83.6 & $\underline{85.2}$ & 83.1 & 83.4 & $\underline{84.1}$ & 83.1 & 83.3 \\
\hline ES & 75.9 & 76.8 & $\underline{78.2}$ & 78.0 & 75.2 & 76.9 & 78.4 & $\underline{79.1}$ & 78.2 & 77.3 & 78.2 & 78.1 & $\underline{78.5}$ \\
\hline ET & 66.1 & 66.2 & 66.6 & $\underline{67.2}$ & 65.9 & 66.6 & 67.7 & 68.4 & 68.3 & 66.7 & $\underline{68.3}$ & 68.2 & 68.0 \\
\hline $\mathbf{E U}$ & 47.2 & 46.3 & 47.7 & $\underline{49.0}$ & 44.4 & 46.4 & 48.7 & 49.2 & $\underline{49.4}$ & 46.1 & 49.7 & 48.7 & $\underline{50.3}$ \\
\hline FR & 83.9 & 83.9 & 84.2 & $\overline{84.4}$ & 83.4 & 83.9 & $\underline{84.7}$ & 84.1 & $\overline{84.6}$ & 83.6 & 83.4 & $\underline{84.1}$ & $\underline{84.1}$ \\
\hline GL & 66.3 & 65.6 & 67.3 & $\underline{68.2}$ & 63.5 & 66.1 & 69.7 & 69.0 & $\underline{70.2}$ & 65.3 & 69.3 & 68.7 & $\underline{70.2}$ \\
\hline HR & 72.3 & 72.7 & 73.9 & $\underline{74.0}$ & 72.2 & 72.8 & 74.3 & 74.2 & $\underline{74.5}$ & 72.9 & 74.5 & 74.1 & $\underline{74.8}$ \\
\hline HU & 67.6 & 68.6 & $\underline{70.7}$ & 70.5 & 67.7 & 68.3 & 71.5 & 71.4 & $\underline{72.1}$ & 68.8 & 72.0 & 71.1 & $\underline{72.1}$ \\
\hline IT & 77.7 & $\underline{78.9}$ & 78.7 & 78.8 & 77.1 & 78.8 & 79.3 & 79.4 & $\overline{79.1}$ & 78.5 & $\underline{79.7}$ & 79.5 & $\overline{79.5}$ \\
\hline JA & 61.9 & 62.3 & 67.1 & $\underline{67.9}$ & 65.0 & 62.4 & 68.9 & 68.3 & $\underline{69.5}$ & 62.3 & $\underline{69.0}$ & 67.1 & 68.4 \\
\hline KO & 64.2 & 63.6 & 64.8 & 64.5 & $\underline{64.9}$ & 63.6 & 65.5 & $\underline{65.7}$ & 65.9 & 63.4 & 64.8 & $\underline{65.6}$ & 65.1 \\
\hline NL & 59.2 & 59.8 & $\underline{60.5}$ & $\underline{60.5}$ & $\overline{58.3}$ & 59.7 & 61.6 & $\overline{61.2}$ & $\underline{62.0}$ & 59.8 & 61.2 & 61.0 & $\underline{61.4}$ \\
\hline SL & 68.4 & 67.2 & $\underline{68.9}$ & 68.6 & 67.0 & 67.4 & $\underline{69.1}$ & 67.9 & 69.0 & 67.8 & 68.4 & 69.5 & $\underline{69.6}$ \\
\hline ZH & 51.6 & 52.0 & 55.9 & $\underline{56.2}$ & $\underline{56.2}$ & 52.2 & 56.6 & $\underline{56.8}$ & 56.5 & 52.5 & $\underline{56.4}$ & 56.0 & 55.9 \\
\hline Avg. & 65.7 & 65.8 & 67.7 & $\underline{68.0}$ & 66.2 & 65.9 & 68.7 & 68.6 & $\underline{68.8}$ & 66.0 & 68.6 & 68.3 & $\underline{68.7}$ \\
\hline
\end{tabular}

Table 1: We report F-1 performance on the XL-WSD dataset. Avg. is the micro-average across all languages but English. +MLM is the baseline model XLM-R which we continued training with the MLM objective only, whereas $+\mathbf{T},+\mathbf{C},+\mathbf{R}$ are the Token, Concat CLS and Replace CLS models, respectively.

\begin{tabular}{|c|c|c|c|c|c|c|c|c|c|c|c|c|c|c|}
\hline & & BG & DA & ET & FA & HR & JA & KO & NL & $\mathbf{Z H}$ & DE & FR & IT & Avg. \\
\hline \multirow{4}{*}{\multicolumn{2}{|c|}{$\begin{array}{l}\text { XLM-R } \\
\text { +MLM (Wiki EN) } \\
\text { +MLM (Wiki 15) } \\
\text { +MLM (Wiki 100) }\end{array}$}} & 61.8 & 65.2 & 62.6 & 65.8 & 66.9 & 61.7 & 65.6 & 69.2 & 68.3 & 61.1 & 58.8 & 62.2 & 64.1 \\
\hline & & 63.0 & 69.9 & 69.7 & 73.6 & 71.3 & 63.7 & 69.5 & 72.4 & 71.5 & 65.1 & 62.3 & 62.5 & 67.9 \\
\hline & & 64.1 & 67.8 & 68.5 & 73.0 & 70.8 & $\frac{0.1}{62.7}$ & 66.8 & 72.9 & 69.8 & 64.1 & 61.5 & 64.5 & $\frac{17.2}{67.2}$ \\
\hline & & 65.5 & 67.9 & 68.5 & 76.3 & 69.1 & 60.8 & 71.1 & 70.5 & 68.3 & 61.7 & 59.7 & 61.2 & 66.7 \\
\hline \multirow{3}{*}{ Wiki EN } & $+T$ & 65.3 & 69.6 & 65.6 & 77.4 & 69.4 & 63.2 & 67.9 & 72.6 & 70.5 & 65.4 & 62.4 & 64.2 & 67.8 \\
\hline & $+\mathrm{C}$ & 66.6 & $\overline{69.0}$ & 68.7 & $\overline{74.9}$ & 74.3 & 65.9 & 69.5 & 72.9 & 70.8 & 67.1 & 63.4 & 66.6 & 69.1 \\
\hline & $+R$ & $\underline{68.4}$ & 68.4 & 69.0 & 75.4 & 73.0 & 65.3 & $\frac{68.4}{6}$ & 73.0 & 69.6 & 66.3 & 62.4 & $\frac{60.9}{64.9}$ & 68.7 \\
\hline \multirow{3}{*}{ Wiki 15} & $+T$ & 64.6 & 67.5 & 64.1 & 75.8 & 68.9 & 62.7 & 71.0 & 70.3 & 67.2 & 63.8 & 61.6 & 65.0 & 66.9 \\
\hline & $+\mathrm{C}$ & 65.0 & 69.5 & 68.7 & $\frac{75.0}{75.3}$ & 69.6 & 64.3 & 69.9 & 73.4 & 70.1 & 65.6 & 61.9 & $\frac{0}{62.5}$ & 68.0 \\
\hline & $+\mathbf{R}$ & 67.4 & 68.0 & 64.4 & 73.3 & 72.1 & 63.4 & 65.1 & 69.5 & 67.1 & 63.3 & $\frac{1.8}{59.8}$ & 61.7 & 66.2 \\
\hline \multirow{3}{*}{ Wiki 100} & $+T$ & 66.7 & 69.7 & 70.5 & 78.5 & 67.9 & $\underline{64.8}$ & 72.3 & 74.3 & 70.9 & 67.2 & 64.0 & 65.7 & 69.4 \\
\hline & $+\mathrm{C}$ & $\overline{61.1}$ & 64.1 & $\overline{65.6}$ & $\overline{71.3}$ & 66.9 & $\overline{60.1}$ & $\overline{68.0}$ & $\overline{67.9}$ & 66.9 & $\overline{59.5}$ & $\overline{57.9}$ & $\overline{59.1}$ & $\overline{64.0}$ \\
\hline & $+\mathbf{R}$ & 65.0 & 70.3 & 68.2 & 73.0 & 72.1 & 62.0 & 68.5 & 71.7 & 72.3 & 65.3 & 61.8 & 63.2 & 67.8 \\
\hline
\end{tabular}

Table 2: Accuracy scores on the crosslingual Word-in-Context (XL-WiC) test set.

15 languages tends to slightly outperform training on all 100 languages on XL-WSD, but on XL-WiC results with our best models trained on 100 languages outperforms all other configurations most of the time by a reasonable margin. These results corroborate our hunch that the intermediate task injects semantic knowledge within the neural model.

In Table 3, we confirm that our models preserve the sentence-level comprehension capabilities of the underlying XLM-R architecture and that it performs either comparably or favourably to the baselines in the XTREME benchmark, across target tasks and languages.
Training on the English Wikipedia only can be surprisingly effective at times (Tables 2 and 3), and training on 100 languages shows more consistent improvements only on XL-WiC but fails to lead to similar improvements on other tasks. We note that performance on XL-WSD is similar when using 15 or 100 languages, while our evaluation using XTREME shows that performance is slightly worse when using 100 languages compared to using 15 languages only. We conjecture this could be due to the fact we finetune only the last two layers of XLM-R (see Appendix B), so the model retains most of the multilingual knowledge it learned dur- 


\begin{tabular}{|c|c|c|c|c|c|c|c|c|c|}
\hline & & $\begin{array}{c}\text { XNLI } \\
a c c .\end{array}$ & $\begin{array}{c}\text { PAWS-X } \\
a c c .\end{array}$ & $\begin{array}{c}\text { POS } \\
F 1\end{array}$ & $\begin{array}{c}\text { NER } \\
F 1\end{array}$ & $\begin{array}{l}\text { XQuAD } \\
F 1 / E M\end{array}$ & $\begin{array}{c}\text { MLQA } \\
F 1 / E M\end{array}$ & $\begin{array}{l}\text { TyDiQA } \\
F 1 / E M\end{array}$ & Avg. \\
\hline \multicolumn{2}{|c|}{ Hu et al. (2020) } & 79.2 & 86.4 & 72.6 & 65.4 & $76.6 / 60.8$ & $71.6 / 53.2$ & $65.1 / 45.0$ & 70.1 \\
\hline \multirow{4}{*}{\multicolumn{2}{|c|}{$\begin{array}{l}\text { XLM-R (Ours) } \\
\text { +MLM (Wiki EN) } \\
\text { +MLM (Wiki 15) } \\
\text { +MLM (Wiki 100) }\end{array}$}} & 78.6 & 87.9 & 76.1 & 64.0 & $71.7 / 56.3$ & $70.3 / 50.0$ & $72.6 / 57.0$ & 70.8 \\
\hline & & 79.1 & 87.9 & 76.4 & 62.3 & $70.6 / 55.2$ & $\overline{69.3} / 50.0$ & $72.7 / 56.8$ & 70.4 \\
\hline & & $\underline{79.3}$ & 88.7 & $\overline{75.7}$ & 64.4 & $\underline{71.8} / \underline{56.8}$ & $70.2 / \underline{50.6}$ & $72.6 / 56.7$ & 71.1 \\
\hline & & $\overline{79.2}$ & $\overline{88.0}$ & 76.0 & $\overline{63.4}$ & $\overline{71.5} / \overline{56.5}$ & $70.1 / \overline{50.5}$ & $\underline{73.5} / \underline{57.4}$ & 70.9 \\
\hline \multirow{3}{*}{ Wiki EN } & $+\mathbf{T}$ & 78.7 & 88.3 & 77.3 & 63.6 & $70.8 / 55.6$ & $69.8 / \underline{50.4}$ & $73.2 / 57.0$ & 70.9 \\
\hline & $+\mathrm{C}$ & 79.0 & 87.9 & $\overline{76.9}$ & 63.7 & $71.5 / 55.7$ & $70.3 / \overline{50.0}$ & $\overline{73.0} / 57.2$ & 70.9 \\
\hline & $+\mathbf{R}$ & $\overline{78.7}$ & $\underline{88.6}$ & 76.9 & $\underline{64.4}$ & $\overline{71.1} / \underline{\underline{55.8}}$ & $\overline{69.6} / 50.1$ & $72.7 / \overline{57.0}$ & 71.0 \\
\hline \multirow{3}{*}{ Wiki 15} & $+T$ & 79.0 & 88.1 & 77.2 & 64.1 & $71.3 / 56.5$ & $70.4 / \mathbf{5 0 . 6}$ & $73.4 / 57.8$ & 71.2 \\
\hline & $+\mathrm{C}$ & $\underline{79.2}$ & $\underline{88.4}$ & $\underline{77.3}$ & 64.7 & $\underline{72.1} / \underline{56.9}$ & $\underline{\mathbf{7 0 . 8}} / 50.5$ & $\overline{73.2} / 57.3$ & $\underline{71.4}$ \\
\hline & $+\mathbf{R}$ & $\overline{79.1}$ & $\overline{88.3}$ & $\overline{76.7}$ & $\overline{64.7}$ & $\overline{71.5} / \overline{56.4}$ & $\overline{70.3} / \underline{\mathbf{5 0 . 6}}$ & $72.8 / 56.7$ & $\overline{71.1}$ \\
\hline \multirow{3}{*}{ Wiki 100} & $+T$ & 78.6 & 88.8 & 76.9 & 64.8 & $71.7 / 56.0$ & $70.1 / 50.0$ & $72.7 / 56.9$ & 71.1 \\
\hline & $+\mathrm{C}$ & 78.6 & 88.6 & 77.6 & 62.1 & $71.2 / 56.4$ & $69.9 / 50.0$ & $73.2 / 57.4$ & 70.9 \\
\hline & $+\mathbf{R}$ & 78.8 & 87.6 & 76.7 & 64.2 & $71.1 / 56.1$ & $69.9 / 50.3$ & $73.1 / 57.4$ & 70.9 \\
\hline
\end{tabular}

Table 3: Results on different target tasks of the XTREME benchmark.

ing pretraining (Liu et al., 2019; Hao et al., 2019).

We also hypothesise that the English Wikipedia size (in number of words) and quality (in coverage of our hyperlink vocabulary) may also be a reason why training solely on English already brings large gains in transfer to other tasks. For comparison, the English Wikipedia is the one with the most data, i.e., about 73M hyperlinks, where the second highest resource language is German with only about $28 \mathrm{M}$ hyperlinks (see Table 4 in Appendix B). Regarding the coverage of our hyperlink vocabulary with $250 \mathrm{k}$ entries, the English Wikipedia covers over $249 \mathrm{k}$ hyperlink types at least 10 times, whereas the second highest coverage is for the French Wikipedia, which covers over 142k hyperlink types at least 10 times. We plan on investigating the effect of the size and coverage of hyperlinks further in future work.

Limitations Finally, we highlight that: (1) We report results using single model runs, therefore we have no estimates of the variance of these models; (2) We lack a more thorough hyperparameter search to further consolidate our results. In both cases, the reason we made such choices is because of the high cost of training large models such as XLM-R large.

\section{Conclusions and Future work}

We presented a multilingual Wikipedia hyperlink prediction intermediate task to improve the pretraining of contextualised word embedding models. We trained three model variants on different sets of languages, finding that injecting multilingual semantic knowledge consistently improves performance on several zero-shot crosslingual tasks. As future work, we plan to devise a solution to allow crosslingual transferability to scale more efficiently with the number of languages. Finally, we will investigate the impact on resource-poor vs resource-rich languages, and the effect of the size and coverage of hyperlinks in model transferability.

\section{Acknowledgments}

We would like to thank Clara Vania and Sam Bowman for comments on early versions of this work, and our three anonymous reviewers for their helpful comments and feedback.

IC has received funding from the European Union's Horizon 2020 research and innovation programme under the Marie Skłodowska-Curie grant agreement No 838188. TP and AR gratefully acknowledge the support of the ERC Consolidator Grants MOUSSE No. 726487, and FoTran No. 771113 under the European Union's Horizon 2020 research and innovation programme. AR also thanks the CSC - IT Center for Science (Finland) for the computational resources.

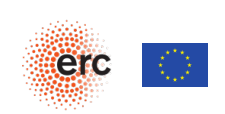

\section{References}

Eneko Agirre, Oier Lopez de Lacalle, Christiane Fellbaum, Shu-Kai Hsieh, Maurizio Tesconi, Monica Monachini, Piek Vossen, and Roxanne Segers. 2010. SemEval-2010 task 17: All-words word sense dis- 
ambiguation on a specific domain. In Proc. of SemEval.

Mikel Artetxe, Sebastian Ruder, and Dani Yogatama. 2020. On the cross-lingual transferability of monolingual representations. In Proceedings of the 58th Annual Meeting of the Association for Computational Linguistics, pages 4623-4637, Online. Association for Computational Linguistics.

Mikel Artetxe and Holger Schwenk. 2019. Massively Multilingual Sentence Embeddings for Zero-Shot Cross-Lingual Transfer and Beyond. Transactions of the ACL 2019.

Giusepppe Attardi. 2015. Wikiextractor. https:// github.com/attardi/wikiextractor.

Laura Benítez, Sergi Cervell, Gerard Escudero, Mònica López, German Rigau, and Mariona Taulé. 1998. Methods and tools for building the Catalan WordNet. Proc. of ELRA Workshop on Language Resources for European Minority Languages.

Michele Bevilacqua and Roberto Navigli. 2020. Breaking through the $80 \%$ glass ceiling: Raising the state of the art in word sense disambiguation by incorporating knowledge graph information. In Proceedings of the 58th Annual Meeting of the Association for Computational Linguistics, pages 2854-2864, Online. Association for Computational Linguistics.

Jonathan H. Clark, Eunsol Choi, Michael Collins, Dan Garrette, Tom Kwiatkowski, Vitaly Nikolaev, and Jennimaria Palomaki. 2020. TyDi QA: A benchmark for information-seeking question answering in typologically diverse languages. Transactions of the Association for Computational Linguistics, 8:454470.

Alexis Conneau, Kartikay Khandelwal, Naman Goyal, Vishrav Chaudhary, Guillaume Wenzek, Francisco Guzmán, Edouard Grave, Myle Ott, Luke Zettlemoyer, and Veselin Stoyanov. 2020. Unsupervised cross-lingual representation learning at scale. In Proceedings of the 58th Annual Meeting of the Association for Computational Linguistics, pages 8440 8451. Association for Computational Linguistics.

Alexis Conneau and Guillaume Lample. 2019. Crosslingual language model pretraining. In $\mathrm{H}$. Wallach, H. Larochelle, A. Beygelzimer, F. d'Alché Buc, E. Fox, and R. Garnett, editors, Advances in Neural Information Processing Systems 32 (NeurIPS), pages 7059-7069. Curran Associates, Inc.

Alexis Conneau, Ruty Rinott, Guillaume Lample, Adina Williams, Samuel Bowman, Holger Schwenk, and Veselin Stoyanov. 2018. XNLI: Evaluating cross-lingual sentence representations. In Proceedings of the 2018 Conference on Empirical Methods in Natural Language Processing, pages 2475-2485, Brussels, Belgium. Association for Computational Linguistics.
Leyang Cui, Sijie Cheng, Yu Wu, and Yue Zhang. 2020. Does bert solve commonsense task via commonsense knowledge? arXiv preprint arXiv:2008.03945.

Jacob Devlin, Ming-Wei Chang, Kenton Lee, and Kristina Toutanova. 2019. BERT: Pre-training of deep bidirectional transformers for language understanding. In Proceedings of the 2019 Conference of the North American Chapter of the Association for Computational Linguistics: Human Language Technologies, Volume 1 (Long and Short Papers), pages 4171-4186, Minneapolis, Minnesota. Association for Computational Linguistics.

Philip Edmonds and Scott Cotton. 2001. SENSEVAL2: Overview. In Proceedings of SENSEVAL-2 Second International Workshop on Evaluating Word Sense Disambiguation Systems, pages 1-5. Association for Computational Linguistics.

Darja Fišer, Jernej Novak, and Tomaž Erjavec. 2012. SloWNet 3.0: development, extension and cleaning. In Proc. of 6th International Global Wordnet Conference.

Edouard Grave, Armand Joulin, Moustapha Cissé, David Grangier, and Hervé Jégou. 2017. Efficient softmax approximation for gpus. In International Conference on Machine Learning (ICML), pages 1302-1310. PMLR.

Xavier Gómez Guinovart. 2011. Galnet: WordNet 3.0 do galego. Linguamática, 3(1).

Yaru Hao, Li Dong, Furu Wei, and Ke Xu. 2019. Visualizing and understanding the effectiveness of BERT. In Proceedings of the 2019 Conference on Empirical Methods in Natural Language Processing and the 9th International Joint Conference on Natural Language Processing (EMNLP-IJCNLP), pages 41434152, Hong Kong, China. Association for Computational Linguistics.

Junjie Hu, Sebastian Ruder, Aditya Siddhant, Graham Neubig, Orhan Firat, and Melvin Johnson. 2020. Xtreme: A massively multilingual multi-task benchmark for evaluating cross-lingual generalization. Proceedings of the 37th International Conference on Machine Learning.

Chu-Ren Huang, Shu-Kai Hsieh, Jia-Fei Hong, YunZhu Chen, I-Li Su, Yong-Xiang Chen, and ShengWei Huang. 2010. Chinese WordNet: Design, Implementation and Application of an Infrastructure for Cross-Lingual Knowledge Processing. Journal of Chinese Information Processing, 24(2).

Hitoshi Isahara, Francis Bond, Kiyotaka Uchimoto, Masao Utiyama, and Kyoko Kanzaki. 2008. Development of the Japanese WordNet. In Sixth International conference on Language Resources and Evaluation. 
Ganesh Jawahar, Benoît Sagot, and Djamé Seddah. 2019. What does bert learn about the structure of language? In Proceedings of the 57th Annual Meeting of the Association for Computational Linguistics, pages $3651-3657$.

Diederik P Kingma and Jimmy Ba. 2015. Adam: A method for stochastic optimization. International Conference on Learning Representations.

Patrick Lewis, Barlas Oguz, Ruty Rinott, Sebastian Riedel, and Holger Schwenk. 2020. MLQA: Evaluating cross-lingual extractive question answering. In Proceedings of the 58th Annual Meeting of the Association for Computational Linguistics, pages 73157330, Online. Association for Computational Linguistics.

Nelson F. Liu, Matt Gardner, Yonatan Belinkov, Matthew E. Peters, and Noah A. Smith. 2019. Linguistic knowledge and transferability of contextual representations. In Proceedings of the 2019 Conference of the North American Chapter of the Association for Computational Linguistics: Human Language Technologies, Volume 1 (Long and Short Papers), pages 1073-1094, Minneapolis, Minnesota. Association for Computational Linguistics.

Ilya Loshchilov and Frank Hutter. 2018. Decoupled weight decay regularization. In International Conference on Learning Representations.

Márton Miháltz, Csaba Hatvani, Judit Kuti, György Szarvas, János Csirik, Gábor Prószéky, and Tamás Váradi. 2008. Methods and Results of the Hungarian WordNet Project. In Proc. of The Fourth Global WordNet Conference.

George A Miller, Claudia Leacock, Randee Tengi, and Ross T Bunker. 1993. A semantic concordance. In Proceedings of the workshop on Human Language Technology, pages 303-308.

Andrea Moro and Roberto Navigli. 2015. SemEval2015 task 13: Multilingual all-words sense disambiguation and entity linking. In Proceedings of the 9th International Workshop on Semantic Evaluation (SemEval 2015), pages 288-297, Denver, Colorado.

Roberto Navigli, David Jurgens, and Daniele Vannella. 2013. SemEval-2013 task 12: Multilingual word sense disambiguation. In Proc. of SemEval.

Roberto Navigli, Kenneth C. Litkowski, and Orin Hargraves. 2007. SemEval-2007 task 07: Coarsegrained English all-words task. In Proc. of SemEval.

Roberto Navigli and Simone Paolo Ponzetto. 2010. BabelNet: Building a very large multilingual semantic network. In Proceedings of the 48th Annual Meeting of the Association for Computational Linguistics, pages 216-225, Uppsala, Sweden. Association for Computational Linguistics.
Joakim Nivre, Mitchell Abrams, Željko Agić, Lars Ahrenberg, Lene Antonsen, Maria Jesus Aranzabe, Gashaw Arutie, Masayuki Asahara, Luma Ateyah, Mohammed Attia, et al. 2018. Universal dependencies 2.2 .

Xiaoman Pan, Boliang Zhang, Jonathan May, Joel Nothman, Kevin Knight, and Heng Ji. 2017. Crosslingual name tagging and linking for 282 languages. In Proceedings of the 55th Annual Meeting of the Association for Computational Linguistics (Volume 1: Long Papers), pages 1946-1958, Vancouver, Canada. Association for Computational Linguistics.

Tommaso Pasini, Alessandro Raganato, and Roberto Navigli. 2021. XL-WSD: An extra-large and crosslingual evaluation framework for word sense disambiguation. In Proceedings of the AAAI Conference on Artificial Intelligence (AAAI).

Bolette S. Pedersen, Sanni Nimb, Jørg Asmussen, Nicolai Hartvig Sørensen, Lars Trap-Jensen, and Henrik Lorentzen. 2009. DanNet: the challenge of compiling a wordnet for Danish by reusing a monolingual dictionary. Language Resources and Evaluation, 43.

Matthew E Peters, Mark Neumann, Robert Logan, Roy Schwartz, Vidur Joshi, Sameer Singh, and Noah A Smith. 2019. Knowledge enhanced contextual word representations. In Proceedings of the 2019 Conference on Empirical Methods in Natural Language Processing and the 9th International Joint Conference on Natural Language Processing (EMNLPIJCNLP), pages 43-54.

Jason Phang, Iacer Calixto, Phu Mon Htut, Yada Pruksachatkun, Haokun Liu, Clara Vania, Katharina Kann, and Samuel Bowman. 2020. English intermediate-task training improves zero-shot crosslingual transfer too. In Proceedings of the 1st Conference of the Asia-Pacific Chapter of the Association for Computational Linguistics and the 10th International Joint Conference on Natural Language Processing, pages 557-575.

Jason Phang, Thibault Févry, and Samuel R Bowman. 2018. Sentence encoders on stilts: Supplementary training on intermediate labeled-data tasks. arXiv preprint arXiv:1811.01088.

Mohammad Taher Pilehvar and Jose CamachoCollados. 2019. WiC: the word-in-context dataset for evaluating context-sensitive meaning representations. In Proceedings of the 2019 Conference of the North American Chapter of the Association for Computational Linguistics: Human Language Technologies, Volume 1 (Long and Short Papers), pages 1267-1273, Minneapolis, Minnesota. Association for Computational Linguistics.

Eli Pociello, Antton Gurrutxaga, Eneko Agirre, Izaskun Aldezabal, and German Rigau. 2008. WNTERM: Enriching the MCR with a terminological 
dictionary. In Proceedings of the Sixth International Conference on Language Resources and Evaluation (LREC'08), Marrakech, Morocco. European Language Resources Association (ELRA).

Marten Postma, Emiel van Miltenburg, Roxane Segers, Anneleen Schoen, and Piek Vossen. 2016. Open Dutch WordNet. In Proc. of the Eight Global Wordnet Conference.

Sameer Pradhan, Edward Loper, Dmitriy Dligach, and Martha Palmer. 2007. SemEval-2007 task-17: English lexical sample, SRL and all words. In Proceedings of the Fourth International Workshop on Semantic Evaluations (SemEval-2007), pages 87-92, Prague, Czech Republic. Association for Computational Linguistics.

Yada Pruksachatkun, Phil Yeres, Haokun Liu, Jason Phang, Phu Mon Htut, Alex Wang, Ian Tenney, and Samuel R. Bowman. 2020. jiant: A software toolkit for research on general-purpose text understanding models. In Proceedings of the 58th Annual Meeting of the Association for Computational Linguistics. System Demonstrations, pages 109-117, Online.

Ida Raffaelli, Marko Tadić, Božo Bekavac, and Željko Agić. 2008. Building Croatian WordNet. In Fourth global wordnet conference (gwc 2008).

Colin Raffel, Noam Shazeer, Adam Roberts, Katherine Lee, Sharan Narang, Michael Matena, Yanqi Zhou, Wei Li, and Peter J. Liu. 2020. Exploring the limits of transfer learning with a unified text-totext transformer. Journal of Machine Learning Research, 21(140):1-67.

Alessandro Raganato, Jose Camacho-Collados, and Roberto Navigli. 2017. Word sense disambiguation: A unified evaluation framework and empirical comparison. In Proceedings of the 15th Conference of the European Chapter of the Association for Computational Linguistics: Volume 1, Long Papers, pages 99-110, Valencia, Spain. Association for Computational Linguistics.

Alessandro Raganato, Tommaso Pasini, Jose CamachoCollados, and Mohammad Taher Pilehvar. 2020. XL-WiC: A multilingual benchmark for evaluating semantic contextualization. In Proceedings of the 2020 Conference on Empirical Methods in Natural Language Processing (EMNLP), pages 7193-7206, Online. Association for Computational Linguistics.

Alessandro Raganato and Jörg Tiedemann. 2018. An analysis of encoder representations in transformerbased machine translation. In Proceedings of the 2018 EMNLP Workshop BlackboxNLP: Analyzing and Interpreting Neural Networks for NLP, pages 287-297, Brussels, Belgium.

Anna Rogers, Olga Kovaleva, and Anna Rumshisky. 2021. A primer in bertology: What we know about how bert works. Transactions of the Association for Computational Linguistics, 8:842-866.
Kiril Simov and Petya Osenova. 2010. Constructing of an Ontology-based Lexicon for Bulgarian. In Proc. of LREC.

Benjamin Snyder and Martha Palmer. 2004. The English all-words task. In Proceedings of SENSEVAL3, the Third International Workshop on the Evaluation of Systems for the Semantic Analysis of Text, pages 41-43, Barcelona, Spain. Association for Computational Linguistics.

Yu Sun, Shuohuan Wang, Yukun Li, Shikun Feng, Hao Tian, Hua Wu, and Haifeng Wang. 2020. Ernie 2.0: A continual pre-training framework for language understanding. In Proceedings of the AAAI Conference on Artificial Intelligence, pages 8968-8975.

Kadri Vider and Heili Orav. 2002. Estonian WordNet and Lexicography. In Proc. of the Eleventh International Symposium on Lexicography.

Xiaozhi Wang, Tianyu Gao, Zhaocheng Zhu, Zhiyuan Liu, Juanzi Li, and Jian Tang. 2021. Kepler: A unified model for knowledge embedding and pretrained language representation. TACL.

Wenhan Xiong, Jingfei Du, William Yang Wang, and Veselin Stoyanov. 2020. Pretrained encyclopedia: Weakly supervised knowledge-pretrained language model. In International Conference on Learning Representations.

Ikuya Yamada, Akari Asai, Hiroyuki Shindo, Hideaki Takeda, and Yuji Matsumoto. 2020. LUKE: Deep contextualized entity representations with entityaware self-attention. In Proceedings of the 2020 Conference on Empirical Methods in Natural Language Processing (EMNLP), pages 6442-6454, Online. Association for Computational Linguistics.

Yinfei Yang, Yuan Zhang, Chris Tar, and Jason Baldridge. 2019. PAWS-X: A cross-lingual adversarial dataset for paraphrase identification. In Proceedings of the 2019 Conference on Empirical Methods in Natural Language Processing and the 9th International Joint Conference on Natural Language Processing (EMNLP-IJCNLP), pages 36873692, Hong Kong, China. Association for Computational Linguistics.

Ae-Sun Yoon, Soon-Hee Hwang, Eun-Ryoung Lee, and Hyuk-Chul Kwon. 2009. Construction of Korean WordNet. Journal of KIISE: Software and Applications, 36(1).

Zhengyan Zhang, Xu Han, Zhiyuan Liu, Xin Jiang, Maosong Sun, and Qun Liu. 2019. ERNIE: Enhanced language representation with informative entities. In Proceedings of the 57th Annual Meeting of the Association for Computational Linguistics, pages 1441-1451, Florence, Italy. Association for Computational Linguistics.

Pierre Zweigenbaum, Serge Sharoff, and Reinhard Rapp. 2018. Overview of the third bucc shared task: Spotting parallel sentences in comparable corpora. 
In Proceedings of 11th Workshop on Building and Using Comparable Corpora, pages 39-42.

\section{A Wikipedia Data Details}

We download the Wikipedia dump from January 11, 2020, and preprocess it using the WikiExtractor script (Attardi, 2015). We download Wikipedia articles for the following 100 languages as in Conneau and Lample (2019): ${ }^{6}$ af, als, am, ang, an, ar, arz, ast, az, bar, be, bg, bn, br, bs, ca, ceb, ckb, cs, cy, $d a, d e, e l$, en, eo, es, et, eu, fa, fi,fr,fy, ga, gan, $g l$, gu, he, hi, hr, hu, hy, ia, id, is, it, ja, jv, ka, kk, kn, ko, ku, la, lb, lt, lv, mk, ml, mn, mr, ms, my, nds, ne, nl, nn, no, oc, pl, pt, ro, ru, scn, sco, sh, si, simple, $s k, s l, s q, s r, s v, s w, t a, t e, t h, t l, t r, t t, u k, u r, u z, v i$, war, wuu, yi, zh_classical, zh, zh_min_nan, zh_yue.

Language sets used for training We finetune MMLM models on the Wikipedia hyperlink prediction task using articles in different sets of languages to investigate the impact of multilingualism. Wiki EN includes only articles in English (en); Wiki 15 includes articles in bg, da, de, en, es, et, eu, $f a, f r, h r, i t, j a, k o, n l, z h$; finally, Wiki 100 includes articles in all 100 languages listed above.

Rationale Wiki $E N$ is a monolingual albeit resource-rich baseline. In Wiki 15, we explore the impact of including languages with different amounts of data and from a mixture of different language families. In Wiki 100, we wish to see if going massively multilingual has a noticeable impact on our models' crosslingual transferability.

Hyperlink extraction We use BabelNet (Navigli and Ponzetto, 2010) - a large multilingual knowledge base comprising WordNet, Wikipedia, and many other resources - to map Wikipedia articles in different languages about the same subject onto unique identifiers. For instance, all "computer science" articles (e.g., Ciencias de la computación in Spanish, Computer science in English, Informatik in German, etc.) are mapped to the same identifier $h_{t}$, in this case bn:00021494n. ${ }^{7}$ After each article is mapped to a single identifier, we create prediction targets for every hyperlink by using the identifier of its referenced article. For example, in Figure 3 the text "algorithmic processes" $\left(x_{n: k}\right)$

\footnotetext{
${ }^{6}$ https://github.com/facebookresearch/ XLM

${ }^{7}$ https: / / babelnet. org/synset?word=bn: $00021494 \mathrm{n} \& \mathrm{lang}=\mathrm{EN}$
}

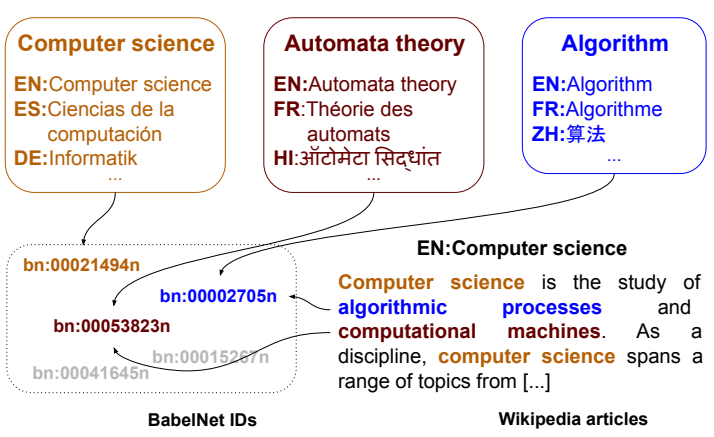

Figure 3: We show Wikipedia articles in different languages, which topics include computer science, automata theory, and algorithm, being mapped to BabelNet IDs. Articles on the same topic, regardless of their language, are mapped to the same identifier. Bottom-right: we show a part of the English article on Computer science and show two example hyperlinks and their targets $h_{t}$.

refers to the article "Algorithm", 8 which is mapped to the ID bn: $00002705 n^{9}\left(h_{t}\right)$.

In Table 4 we show detailed per-language statistics for the Wikipedia data used in our experiments, including the size of the datasets and the number of hyperlinks appearing in the articles (this count already includes only the hyperlinks in our hyperlinks vocabulary of $250 \mathrm{k}$ types).

\section{B Hyperparameters, Training Procedure and Model Architectures}

We use XLM-R-large (Conneau et al., 2020), which has an encoder with 24 layers and a hidden state size 1024. We finetune XLM-R-large using AdamW (Kingma and Ba, 2015; Loshchilov and Hutter, 2018) with learning rate 0.00005 , no weight decay, and batch size 16 . We train on minibatches with maximum sequence length 256 , gradient norm set to 1.0 , and for $300 \mathrm{k}$ model updates. When finetuning XLM-R on Wikipedia hyperlink prediction, we only update the last two layers of the model.

Training data sampling We sample batches of training data from each of the languages available, i.e., depending on the experiment these can be English only, 15 languages, or 100 languages. We sample with probability $r_{l}=\frac{\min \left(e_{l}, K\right)}{\sum\left(\min \left(e_{l}, K\right)\right.}$, where $e_{l}$ is the number of examples per language $l$ and the constant $K=2^{17}$ leads to sampling more often

\footnotetext{
${ }^{8}$ https://en.wikipedia.org/wiki/ Algorithm

${ }^{9}$ https: / / babelnet. org/ synset?word=bn: $00002705 \mathrm{n} \& \mathrm{lang}=\mathrm{EN}$
} 
from resource-poor languages (Raffel et al., 2020).

Adaptive softmax We collect hyperlink targets $h_{t}$ from across Wikipedia articles in all the 100 languages available, sort these hyperlinks from most to least frequent, and keep only the top 250k hyperlink targets $h_{t}$. Since hyperlink frequencies follow a natural Zipfian distribution, we use the adaptive softmax activation (Grave et al., 2017) to predict hyperlinks. We bin hyperlink mentions from most to least frequent, i.e. the most frequent $h_{t}$ is ranked 1 st and the least frequent $h_{t}$ is ranked 250k-th. We use five bins, which include hyperlinks with ranks in the following intervals: $[1,10 \mathrm{k}]$, (10k, 40k], (40k, 50k], (50k, 70k], (70k, 250k].

The adaptive softmax activation is efficient to compute because: (1) we use one matrix multiplication for each bin, drastically reducing the number of parameters; and (2) the latter bins are only computed in case there is at least one entry in the minibatch with a target in that bin. The five-weight matrices that parameterise each bin in our adaptive softmax layer have sizes: hdim $\times 10,000$, hdim $\times 30,000$, hdim $\times 10,000$, hdim $\times 20,000$, hdim $\times 180,000$, respectively. Since bins are constructed so that the least frequent hyperlinks are added to the latter bins, we rarely need to compute them. This is especially important in case of the last bin, which is the most costly to compute (and is rarely used).

\section{B.1 Model Architectures}

We refer the reader for the mathematical notation in Section 2 Approach. The Wikipedia hyperlink prediction head for a single hyperlink using each of our models is shown below. Token is computed in Equation 1.

$$
p\left(x_{i}=k\right) \propto \text { AdaptiveSoftmax }_{k}\left(W_{t} \cdot \boldsymbol{x}_{i}+b_{t}\right),
$$

where AdaptiveSoftmax ${ }_{k}$ computes the probability of the hyperlink target $h_{t}=k, \boldsymbol{x}_{n: k}$ is a hyperlink consisting of words $\left\{\boldsymbol{x}_{n}, \cdots, \boldsymbol{x}_{k}\right\}$, and $W_{t}$ and $b_{t}$ are trained parameters.

Concat CLS is computed in Equation 2.

$$
\begin{gathered}
p\left(x_{i}=k\right) \propto \\
\text { AdaptiveSoftmax }_{k}\left(W_{c} \cdot\left[\boldsymbol{x}_{i} ; \boldsymbol{x}_{\mathrm{CLS}}\right]+b_{c}\right),
\end{gathered}
$$

where AdaptiveSoftmax ${ }_{k}$ computes the probability of the hyperlink target $h_{t}=k, \boldsymbol{x}_{n: k}$ is a hyperlink consisting of words $\left\{\boldsymbol{x}_{n}, \cdots, \boldsymbol{x}_{k}\right\}$, and $W_{c}$ and $b_{c}$ are trained parameters.
Replace CLS is computed in Equation 3.

$$
\begin{aligned}
\boldsymbol{x} & =\operatorname{sample}\left(\boldsymbol{x}_{i}, \boldsymbol{x}_{\mathrm{CLS}}\right), \\
p\left(x_{i}=k\right) & \propto \operatorname{AdaptiveSoftmax}_{k}\left(W_{r} \cdot \boldsymbol{x}+b_{r}\right),
\end{aligned}
$$

where sample $(a, b)$ samples $a$ or $b$ with probability 0.9 and 0.1 , respectively; AdaptiveSoftmax ${ }_{k}$ computes the probability of the hyperlink target $h_{t}=k, \boldsymbol{x}_{n: k}$ is a hyperlink consisting of words $\left\{\boldsymbol{x}_{n}, \cdots, \boldsymbol{x}_{k}\right\}$, and $W_{r}$ and $b_{r}$ are trained parameters.

\section{B.1.1 XL-WSD}

We freeze the pretrained MMLM model weights and simply add a trained classification head on top of the pretrained MMLM. We compute representations for each subword as the sum of the last 4 layers of the model, and for each word as the average of its subword representations (Bevilacqua and Navigli, 2020).

\section{B.1.2 XL-WiC}

We follow Raganato et al. (2020) and add a binary classification head on top of the pretrained MMLM model, which takes as input the concatenation of the target words' embedding in the two contexts. We use the output of the 24-th layer as the target words' representation.

\section{B.1.3 XTREME}

We use the Jiant library (Pruksachatkun et al., 2020) to carry out the evaluation on XTREME. We use the output of the 24-th layer as the input token representations so as to better measure the impact of our intermediate training on the XTREME tasks.

\section{B.2 XTREME Sentence Retrieval Tasks}

BUCC (Zweigenbaum et al., 2018), and Tatoeba (Artetxe and Schwenk, 2019) are two unsupervised tasks requiring, given a sentence in a language $L$ to retrieve its closest sentence in another language $L^{\prime}$. XTREME baselines use the average of the 14-th layer outputs to represent the sentence. ${ }^{10}$ Since our intermediate training procedure only tunes the last two layers, the output of the 14-th layer would be the exact same of the plain XLM-R baseline. For this reason, we did not report the results in both tasks.

\footnotetext{
${ }^{10}$ https://github.com/nyu-mll/jiant/ blob/master/guides/tasks/task_specific. md
} 


\begin{tabular}{|c|c|c|c|c|c|c|c|}
\hline $\begin{array}{l}\text { Language } \\
\text { Code }\end{array}$ & Language & $\begin{array}{r}\text { \# training } \\
\text { links }\end{array}$ & size & $\begin{array}{l}\text { Language } \\
\text { Code }\end{array}$ & Language & $\begin{array}{r}\text { \# training } \\
\text { links }\end{array}$ & size \\
\hline $\mathrm{AF}$ & Afrikaans & 524,682 & $37 \mathrm{M}$ & $\mathrm{KO}$ & Korean & $2,955,253$ & $191 \mathrm{M}$ \\
\hline ALS & Tosk Albanian & 203,333 & $13 \mathrm{M}$ & KU & Kurdish & 91,047 & $4,6 \mathrm{M}$ \\
\hline AM & Amharic & 35,586 & $2,1 \mathrm{M}$ & LA & Latin & 791,760 & $29 \mathrm{M}$ \\
\hline ANG & Anglo-Saxon & 315,250 & $530 \mathrm{~K}$ & LB & Luxembourgish & 224,604 & $13 \mathrm{M}$ \\
\hline AN & Aragonese & 8,376 & $12 \mathrm{M}$ & LT & Lithuanian & $1,276,418$ & $76 \mathrm{M}$ \\
\hline $\mathrm{AR}$ & Arabic & $6,342,628$ & $343 \mathrm{M}$ & $\mathrm{LV}$ & Latvian & 748,963 & $46 \mathrm{M}$ \\
\hline ARZ & Egyptian Arabic & $1,738,581$ & $35 \mathrm{M}$ & MK & Macedonian & 983,105 & $63 \mathrm{M}$ \\
\hline AST & Asturian & 971,410 & $77 \mathrm{M}$ & ML & Malayalam & 332,530 & $37 \mathrm{M}$ \\
\hline $\mathrm{AZ}$ & Azerbaijani & 786,016 & $53 \mathrm{M}$ & MN & Mongolian & 90,807 & $6,3 \mathrm{M}$ \\
\hline BAR & Bavarian & 78,614 & $4,4 \mathrm{M}$ & MR & Marathi & 169,347 & $13 \mathrm{M}$ \\
\hline $\mathrm{BE}$ & Belarusian & $1,138,871$ & $82 \mathrm{M}$ & MS & Malay & $1,242,850$ & $54 \mathrm{M}$ \\
\hline BG & Bulgarian & $2,340,267$ & $158 \mathrm{M}$ & MY & Burmese & 48,285 & $5,9 \mathrm{M}$ \\
\hline $\mathrm{BN}$ & Bengali & 549,982 & $56 \mathrm{M}$ & NDS & Low Saxon & 168,053 & $12 \mathrm{M}$ \\
\hline BR & Breton & 318,303 & $15 \mathrm{M}$ & $\mathrm{NE}$ & Nepali & 66,667 & $5,8 \mathrm{M}$ \\
\hline BS & Bosnian & 596,758 & $34 \mathrm{M}$ & NL & Dutch & $10,647,696$ & $551 \mathrm{M}$ \\
\hline CA & Catalan & $6,180,563$ & $395 \mathrm{M}$ & NN & Norwegian Nynorsk & 983,245 & $54 \mathrm{M}$ \\
\hline CEB & Cebuano & $15,029,079$ & $178 \mathrm{M}$ & NO & Norwegian & $4,095,644$ & $227 \mathrm{M}$ \\
\hline CKB & Central Kurdish & 88,593 & $6,1 \mathrm{M}$ & $\mathrm{OC}$ & Occitan & 562,718 & $22 \mathrm{M}$ \\
\hline $\mathrm{CS}$ & Czech & $4,697,945$ & $341 \mathrm{M}$ & PL & Polish & $10,753,690$ & $685 \mathrm{M}$ \\
\hline CY & Welsh & 561,936 & $23 \mathrm{M}$ & PT & Portuguese & $10,065,298$ & $581 \mathrm{M}$ \\
\hline DA & Danish & $2,273,079$ & $135 \mathrm{M}$ & RO & Romanian & $2,376,428$ & $129 \mathrm{M}$ \\
\hline $\mathrm{DE}$ & German & $28,064,840$ & $2,1 \mathrm{G}$ & RU & Russian & $15,691,268$ & $1,4 \mathrm{G}$ \\
\hline EL & Greek & $1,611,904$ & $166 \mathrm{M}$ & $\mathrm{SCN}$ & Sicilian & 64,902 & $3,4 \mathrm{M}$ \\
\hline $\mathrm{EN}$ & English & $73,084,305$ & $4,9 \mathrm{G}$ & $\mathrm{SCO}$ & Scots & 174,304 & $9,7 \mathrm{M}$ \\
\hline EO & Esperanto & $2,330,837$ & $110 \mathrm{M}$ & $\mathrm{SH}$ & Serbo-Croatian & $3,076,574$ & $113 \mathrm{M}$ \\
\hline $\mathrm{ES}$ & Spanish & $19,125,611$ & $1,2 \mathrm{G}$ & SI & Sinhala & 26,321 & $3,5 \mathrm{M}$ \\
\hline ET & Estonian & $1,417,295$ & $82 \mathrm{M}$ & SIMPLE & Simple English & $1,260,400$ & $57 \mathrm{M}$ \\
\hline EU & Basque & $1,743,033$ & $71 \mathrm{M}$ & SK & Slovak & $1,455,414$ & $84 \mathrm{M}$ \\
\hline FA & Persian & $3,429,725$ & $185 \mathrm{M}$ & SL & Slovenian & $1,343,091$ & $78 \mathrm{M}$ \\
\hline FI & Finnish & $3,748,928$ & $252 \mathrm{M}$ & SQ & Albanian & 280,756 & $18 \mathrm{M}$ \\
\hline FR & French & $23,415,178$ & $1,5 \mathrm{G}$ & SR & Serbian & $3,218,656$ & $194 \mathrm{M}$ \\
\hline FY & Western Frisian & 423,234 & $26 \mathrm{M}$ & SV & Swedish & $21,025,833$ & $475 \mathrm{M}$ \\
\hline GA & $\mathrm{Ga}$ & 183,946 & $11 \mathrm{M}$ & SW & Swahili & 317,669 & $11 \mathrm{M}$ \\
\hline GAN & Gan Chinese & 8,742 & $425 \mathrm{~K}$ & $\mathrm{TA}$ & Tamil & 691,010 & $54 \mathrm{M}$ \\
\hline GL & Galician & $1,632,786$ & $108 \mathrm{M}$ & TE & Telugu & 307,488 & $26 \mathrm{M}$ \\
\hline GU & Gujarati & 256,284 & $6,6 \mathrm{M}$ & TH & Thai & 862,265 & $82 \mathrm{M}$ \\
\hline $\mathrm{HE}$ & Hebrew & $6,256,536$ & $396 \mathrm{M}$ & $\mathrm{TL}$ & Tagalog & 218,323 & $13 \mathrm{M}$ \\
\hline HI & Hindi & 546,648 & $45 \mathrm{M}$ & TR & Turkish & $2,336,668$ & $150 \mathrm{M}$ \\
\hline HR & Croatian & $1,825,455$ & $112 \mathrm{M}$ & $\mathrm{TT}$ & Tatar & 499,022 & $15 \mathrm{M}$ \\
\hline $\mathrm{HU}$ & Hungarian & $3,785,965$ & $275 \mathrm{M}$ & UK & Ukrainian & $7,949,672$ & $562 \mathrm{M}$ \\
\hline HY & Armenian & $1,639,954$ & $124 \mathrm{M}$ & UR & Urdu & 526,498 & $29 \mathrm{M}$ \\
\hline IA & Interlingua & 51,882 & $2,4 \mathrm{M}$ & UZ & Uzbek & 308,536 & $9,3 \mathrm{M}$ \\
\hline ID & Indonesian & $3,504,017$ & $159 \mathrm{M}$ & VI & Vietnamese & $4,877,318$ & $221 \mathrm{M}$ \\
\hline IS & Icelandic & 252,888 & $17 \mathrm{M}$ & WAR & Waray & $4,738,778$ & $46 \mathrm{M}$ \\
\hline IT & Italian & $15,407,079$ & $1011 \mathrm{M}$ & WUU & Wu Chinese & 47,388 & $4,4 \mathrm{M}$ \\
\hline JA & Japanese & $13,318,170$ & $949 \mathrm{M}$ & YI & Yiddish & 85,374 & $5,1 \mathrm{M}$ \\
\hline JV & Javanese & 213,822 & $11 \mathrm{M}$ & ZH CLASSICAL & Classical Chinese & $7,654,040$ & $2,9 \mathrm{M}$ \\
\hline KA & Georgian & 894,180 & $70 \mathrm{M}$ & $\mathrm{ZH}$ & Chinese & 38,104 & $499 \mathrm{M}$ \\
\hline KK & Kazakh & 660,065 & $41 \mathrm{M}$ & ZH MIN NAN & Min Nan & $1,101,622$ & $12 \mathrm{M}$ \\
\hline $\mathrm{KN}$ & Kannada & 114,724 & $16 \mathrm{M}$ & ZH YUE & Cantonese & 344,432 & $17 \mathrm{M}$ \\
\hline
\end{tabular}

Table 4: Data statistics: total number of hyperlinks appearing in articles in Wikipedia in a given language, and size of the dataset for each language. $\mathrm{K}$ stands for kilobyte, $\mathrm{M}$ for megabyte, and $\mathrm{G}$ for gigabyte. 